\title{
On the Breakdown of the Ehrenfest Method for Molecular Dynamics on Surfaces
}

\author{
Ignacio Loaiza and Artur F. Izmaylov \\ Chemical Physics Theory Group, Department of Chemistry, \\ University of Toronto, Toronto, Ontario M5S3H6, Canada and \\ Department of Physical and Environmental Sciences, \\ University of Toronto Scarborough, Toronto, Ontario, M1C1A4, Canada
}

(Dated: September 12, 2018)

\begin{abstract}
Due to a continuum of electronic states present in periodic systems, the description of molecular dynamics on surfaces poses a serious computational challenge. One of the most used families of approaches in these settings are friction theories, which are based on the Ehrenfest (EH) approach. Yet, a mean-field treatment of electronic degrees of freedom in the EH method makes this approach inaccurate in some cases. Our aim is to clarify when EH breaks down for molecular dynamics on surfaces. Answering this question provides limits of applicability for more approximate friction theories derived from EH. We assess the EH method on one-dimensional, numerically exactly solvable models with a large but finite number of electronic states. Using the Landau-Zener formula and the Massey parameter, an expression that determines when EH breaks down is deduced.
\end{abstract}

\section{INTRODUCTION}

Molecular dynamics in periodic systems in general and on metallic surfaces in particular is complicated by the presence of a continuum of electronic states. This continuum can lead to break-down of the Born-Oppenheimer approximation, which is based on the assumption that the energy separation between electronic states is much larger than the nuclear kinetic energy. This means that a proper treatment of such systems must take into account nonadiabatic effects. The most computationally feasible candidates for simulations in extended systems are mixed quantum-classical (MQC) methods, ${ }^{1-4}$ such as the Ehrenfest (EH) approach, ${ }^{5}$ and Tully's fewest switches surface hopping (SH) algorithm. ${ }^{5-7}$ Yet, due to the continuum of electronic states, $\mathrm{EH}$ and $\mathrm{SH}$ are not straightforwardly applicable to periodic systems. First, the continuum of states must be discretized, ${ }^{8}$ and even then thousands of electronic states can be involved in dynamics, ${ }^{9}$ rendering such calculations computationally challenging.

To avoid explicit treatment of a large number of electronic states, frictional theories were introduced. In these theories, starting from the $\mathrm{EH}$ formalism, the electronic continuum is approximately integrated, ${ }^{10}$ and nuclear trajectories evolve on the adiabatic ground state with an additional frictional force arising from nonadiabatic transitions. The frictional approaches have been extensively studied over the years. ${ }^{10-15}$ The problem with frictional approximations appears when multiple electronic surfaces with very different nuclear dependence become energetically available. This often takes place for different adsorbate states, for example, dynamics of $\mathrm{Cl} / \mathrm{Cl}^{-}$adsorbates on the $\mathrm{Cu}$ (110) surface in scanning tunneling microscope induced reactions. ${ }^{16,17}$ Here, the underlying EH may not be as good as in the case when only one adsorbate state is involved.

Some inaccuracies introduced by frictional approximations were corrected by switching to a collective variable approach. ${ }^{1}$ This approach does not treat electronic DOF fully implicitly but rather reduces their explicit consid- eration to only few (two or three) collective electronic coordinates. However, in the current formulation this approach still cannot surpass the EH method which it is based on. Applying the collective variable idea to the $\mathrm{SH}$ framework would be ideal in cases when several adsorbate states are involved in surface dynamics, since this framework allows for proper account of dynamics on individual electronic states. ${ }^{18}$

Still, EH and EH-based methods are frequently used in modeling surface dynamics, and it is instructive to develop a numerical criterion indicating when going beyond the $\mathrm{EH}$ framework is necessary. This is the main objective of the present work. We begin by reviewing the $\mathrm{EH}$ and $\mathrm{SH}$ methods with an illustration of $\mathrm{EH}$ failure on Tully's extended coupling model in Sec. II A. Using the Landau-Zener (LZ) formula, we deduce a parameter that can indicate when EH dynamics breaks down in Sec. II B. Section III illustrates performance of the new indicator on one-dimensional surface dynamics inspired models.

\section{THEORY}

\section{A. Mixed quantum-classical methods}

To introduce notation and to provide self-sufficient description of methodology we start with a brief reminder of the $\mathrm{EH}$ and $\mathrm{SH}$ methods. ${ }^{5,6,19}$ In both of these methods, nuclear coordinates $\mathbf{R}$ are treated classically and are substituted by corresponding time-dependent functions $\mathbf{R}=\mathbf{R}(t)$. Using this substitution one can formulate the total non-stationary electronic wavefunction as

$$
|\Psi(\mathbf{R}(t), t)\rangle=\sum_{j} c_{j}(t)\left|\phi_{j}[\mathbf{R}(t)]\right\rangle
$$

where $\left|\phi_{j}[\mathbf{R}(t)]\right\rangle$ are eigenfunctions of the electronic Hamiltonian $\hat{H}_{e}[\mathbf{R}(t)], \quad \hat{H}_{e}[\mathbf{R}(t)]\left|\phi_{j}[\mathbf{R}(t)]\right\rangle=$ $E_{j}[\mathbf{R}(t)]\left|\phi_{j}[\mathbf{R}(t)]\right\rangle$, and $E_{j}[\mathbf{R}(t)]$ are corresponding adiabatic PESs. To formulate equations of motion for 
time-dependent coefficients $c_{j}(t)$ the time-dependent Schrödinger equation

$$
i \frac{\partial}{\partial t}|\Psi(\mathbf{R}(t), t)\rangle=\hat{H}_{e}[\mathbf{R}(t)]|\Psi(\mathbf{R}(t), t)\rangle
$$

is projected onto the adiabatic wavefunctions $\left|\phi_{j}[\mathbf{R}(t)]\right\rangle$

$$
\dot{\mathbf{c}}=-\left(i \mathbf{H}_{\mathbf{e}}+\dot{\mathbf{R}} \cdot \boldsymbol{\Gamma}\right) \mathbf{c},
$$

where $\boldsymbol{\Gamma}$ is a matrix with elements $\boldsymbol{\Gamma}_{j k}=$ $\left\langle\phi_{j}[\mathbf{R}] \mid \nabla_{\mathbf{R}} \phi_{k}[\mathbf{R}]\right\rangle \quad$ corresponding to the nonadiabatic coupling (NAC) vectors, and $\mathbf{c}$ is a vector with entries $c_{j}(t)$. In the adiabatic representation, $\mathbf{H}_{\mathbf{e}}$ is a diagonal matrix with elements $\mathbf{H}_{\mathbf{e}, k j}=\left\langle\phi_{k}(\mathbf{R})\left|\hat{H}_{e}(\mathbf{R})\right| \phi_{j}(\mathbf{R})\right\rangle=E_{k}(\mathbf{R}) \delta_{k j}$.

In $\mathrm{EH}$ and $\mathrm{SH}$ methods the nuclear dynamics, $\mathbf{R}(t)$, is governed by Newton equations of motion. For EH, the force is based on the gradient of the averaged electronic energy

$$
\mathbf{F}=-\nabla_{\mathbf{R}}\left\langle\Psi\left|\hat{H}_{e}\right| \Psi\right\rangle .
$$

Using the adiabatic expansion for $|\Psi\rangle$ in Eq. (1) and

$$
\nabla_{\mathbf{R}}\left\langle\phi_{j}\left|\hat{H}_{e}\right| \phi_{k}\right\rangle=\left(E_{k}-E_{j}\right) \boldsymbol{\Gamma}_{j k}+\left\langle\phi_{j}\left|\nabla_{\mathbf{R}} \hat{H}_{e}\right| \phi_{k}\right\rangle
$$

one obtains the nuclear equation of motion in the Ehrenfest method as

$$
M \ddot{\mathbf{R}}=-\sum_{k}\left|c_{k}\right|^{2} \nabla_{\mathbf{R}} E_{k}+\sum_{k \neq j} c_{k}^{*} c_{j}\left(E_{k}-E_{j}\right) \boldsymbol{\Gamma}_{k j} .
$$

This equation can also be derived from the conservation of the total energy $E=\frac{1}{2} M|\dot{\mathbf{R}}|^{2}+\left\langle\Psi\left|\hat{H}_{e}\right| \Psi\right\rangle$.

In $\mathrm{SH}$, nuclear dynamics is governed by forces from a single potential energy surface (PES) at each moment of time

$$
M \ddot{\mathbf{R}}=-\nabla_{\mathbf{R}} E_{j}(\mathbf{R}) .
$$

However, to allow for nonadiabatic dynamics, a probability $P_{j \rightarrow k}$ of changing a PES corresponding to a state $\left|\phi_{j}\right\rangle$ to that of a state $\left|\phi_{k}\right\rangle$ is introduced for every trajectory, a so-called hopping probability,

$$
P_{j \rightarrow k}=\frac{2 \operatorname{Re}\left(c_{j}^{*} c_{k} \dot{\mathbf{R}} \cdot \boldsymbol{\Gamma}_{j k}\right) \Delta t}{\left|c_{j}\right|^{2}},
$$

where $\Delta t$ is a time-step. In both methods an ensemble of trajectories is used to model the quantum nuclear distribution.

Thus, EH and SH have the same electronic dynamic equation Eq.(3), but different nuclear dynamics. These differences in treatments of nuclear DOF can result in significant differences in nuclear dynamics in cases where the following two conditions are satisfied: 1) the coupling region is followed by the region where participating PESs have different slopes, 2) probabilities to find the system on either of participating PESs are similar. A good, illustrative example of these differences is dynamics in the
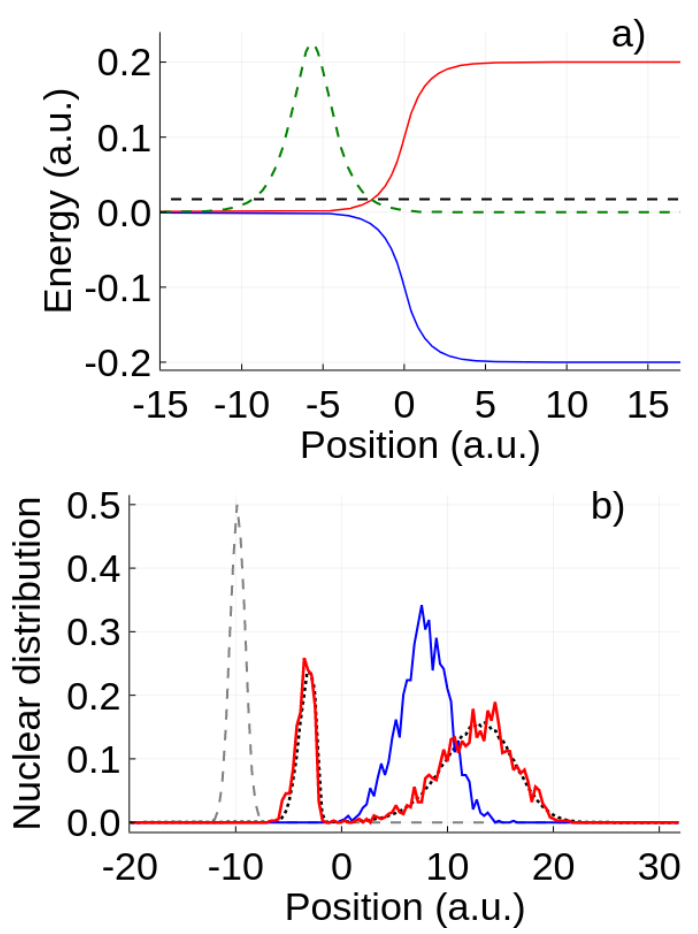

FIG. 1. a) PESs (red and blue solid) for Tully's extended coupling model, ${ }^{6}$ the initial energy (horizontal dashed), and NACs (green dashed). b) Initial (dashed gray, its magnitude is divided by 2) and final nuclear position distributions (blue for $\mathrm{EH}$, red for $\mathrm{SH}$, and black dots for exact quantum). The initial Wigner distribution is centered at $R=-10$ and has average momentum $p=8.5$.

Tully extended coupling model ${ }^{6}$ (Fig. 1a). The difference in PES slopes after the coupling region and the chosen initial energy causes the nuclear wave-packet to split, the part on the excited PES is reflected while that on the ground state PES is transmitted (Fig. 1b). SH gives a nuclear distribution almost indistinguishable from the exact quantum one, while EH fails to capture the branching of the nuclear distribution. For the comparison with the exact quantum dynamics, we used the split operator (SO) method. ${ }^{20}$

\section{B. Ehrenfest break-down indicator}

To provide more quantitative measure for possibility of the Ehrenfest method failure in situations where involved PESs have different slopes, we will use the LZ formula ${ }^{21}$ to estimate the probabilities of finding the system on different PESs. The LZ equation does not require coefficients for the electronic states entering Eq. (8), and therefore, is more convenient for estimates. Of course, use of the LZ expression introduces certain constraints, however, such constraints are generally consistent with considered processes of interest: molecular dynamics of an adsorbate that has at least two different molecular electronic states, whose PESs approach each other in localized nuclear configuration regions. Thus, all models considered in this 


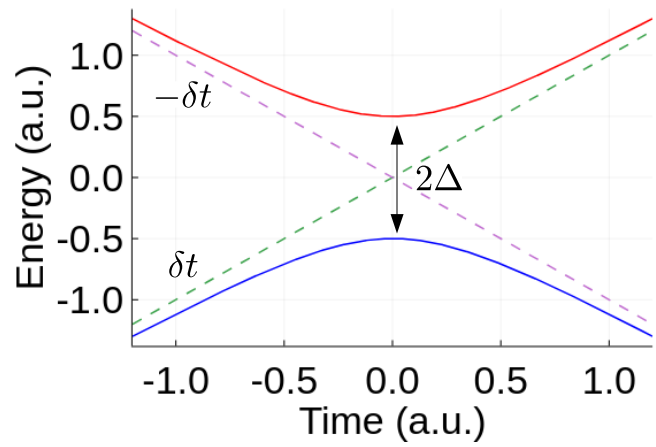

FIG. 2. Landau-Zener crossing model with $\delta=1$ and $\Delta=0.5$. Adiabatic (diabatic) PESs are solid (dashed) lines.

work have nonadiabatic couplings localized near crossings of diabatic potentials.

The LZ potential assumes that the transition region is so small that the energy difference between diabats may be seen as a linear function in time $(2 \delta t)$, while the offdiagonal coupling $\Delta$ is a constant, and is defined, along with all its auxiliary variables, in Appendix A. The LZ formula for calculating the probability of changing from diabatic surface $a$ to $b$ after passing through a diabatic potentials (Fig. 2) crossing point is

$$
P_{b \leftarrow a}=1-\exp \left(\frac{-2 \pi \Delta^{2}}{\dot{R}\left|F_{b}-F_{a}\right|}\right)
$$

where $\dot{R}$ is the nuclear velocity evaluated at the crossing point, and $F_{b}$ and $F_{a}$ are the diabatic forces at that point. In the adiabatic picture, $P_{b \leftarrow a}$ corresponds to the probability of staying on the adiabatic PES that was very similar to the diabatic potential of the initial diabatic state $(a)$ and $(b)$.

The argument of the exponential function is called the Massey parameter ${ }^{22}$

$$
\xi_{\text {dia }}=\frac{2 \pi \Delta^{2}}{\dot{R}\left|F_{b}-F_{a}\right|} .
$$

It can be used as an indicator for adiabatic $(\xi \gg 1)$ or diabatic $(\xi \ll 1)$ behavior. It was originally derived in the diabatic basis, ${ }^{21}$ but it can also be obtained in the adiabatic representation using a perturbative approach. ${ }^{23}$ Also, using the LZ model, it is possible to transform the Massey parameter $\xi_{\text {dia }}$ to the adiabatic representation (see Appendix A for details)

$$
\xi_{\text {adi }}=\frac{\pi\left(E_{2}-E_{1}\right)}{4\left|\left\langle\phi_{1} \mid \partial_{t} \phi_{2}\right\rangle\right|}=\frac{\pi\left(E_{2}-E_{1}\right)}{4 \dot{R}\left|\Gamma_{12}\right|},
$$

where $E_{i}{ }^{\prime}$ 's are adiabatic energies, $\left\langle\phi_{1} \mid \partial_{t} \phi_{2}\right\rangle$ is a timederivative coupling, and $\Gamma_{12}$ is the NAC; all quantities are taken at the diabatic crossing point or at the minimal gap point. The adiabatic formulation is beneficial for analyzing the data from first-principles calculations that do not have underlying diabatic models. Because of this, we will use $\xi \equiv \xi_{\text {adi }}$.
From previous consideration we know that EH will be problematic when probabilities of finding the system on competing pathways are similar, we judiciously consider the Massey parameter $\xi=1$ to be an indicator of this case.

To calculate Massey's parameter from either Eq. (10) or Eq. (11), the value of the nuclear velocity can be estimated from the energy conservation condition. We have two different values for $\dot{R}$ : $\dot{R}_{\text {adi }}$, which assumes a motion on the ground adiabatic state, and $\dot{R}_{\text {dia }}$ for a motion in the first diabatic state. Both velocities would then represent an unperturbed motion in their respective bases. Given an initial total energy $\epsilon$ for a trajectory, the velocities are evaluated as

$$
\dot{R}=\left(\frac{2}{M}(\epsilon-E)\right)^{\frac{1}{2}},
$$

where for $\dot{R}=\dot{R}_{\text {dia }}\left(\dot{R}_{\text {adi }}\right)$ we use $E=E_{a}\left(E_{1}\right)$ at the diabatic crossing point. Since we are working with the adiabatic values, we will be using the velocity estimate using $E=E_{1}$ in Eq. (12).

\section{RESULTS AND DISCUSSION}

To illustrate correlation between our indicator and performance of mixed quantum-classical (MQC) methods we performed simulations with the $\mathrm{EH}, \mathrm{SH}$, and $\mathrm{SO}$ methods on two one-dimensional models. All MQC simulations were run using 2000 trajectories with initial conditions sampled from a Wigner Gaussian distribution with the standard deviation of $1 / \sqrt{2}$ for both nuclear positions and momenta. Dynamics were propagated using the fourthorder Runge-Kutta method for $\approx 121 \mathrm{fs}$, with a time-step of $\Delta t=1.0(0.1)$ a.u. for a sinusoidal (metallic surface) model. In all cases, dynamics were checked to yield converged results with respect to the time-step. The particle mass was set to be of 2000 a.u.

All SH dynamics were done in the adiabatic basis except in the low coupling case shown in Fig. 4c,d. For this case, the motion is highly diabatic, and as discussed in Ref. 24, $\mathrm{SH}$ yields better results in the representation with fewer hops, which is the diabatic representation in this case.

All coding was done using the Julia language, and the code is available at https://github.com/iloaiza/MQC.

\section{A. Sinusoidal model}

A sinusoidal model has a diabatic potential of the form

$$
V(R)=\left[\begin{array}{cc}
A \sin (k R) & \Delta \\
\Delta & -A \sin (k R)
\end{array}\right] .
$$

This potential will serve to model periodic systems, while offering a very simple parametric dependence that allows us to easily explore several coupling regimes by varying $\Delta$ with fixed $A=0.02$ and $k=0.5$. 
Based on particle's initial energy we distinguish the following dynamical regimes in this model: (1) Trapped regime: the initial energy is lower than the adiabatic potential energy barrier height on the ground adiabatic state. This case is trivial since there is no appreciable non-adiabatic dynamics taking place, instead the particle is trapped on the ground surface site. (2) Adiabatic regime: there is enough energy to cross the adiabatic barrier but not for appreciable probability of nonadiabatic transition to the excited state. (3) Nonadiabatic regime: the particle has enough energy to be promoted to the excited state but not enough to cross the excited state barrier. (4) High energy regime: the initial energy is higher than the excited PES barrier. For our investigation only the second, third and fourth regimes are of interest.

a. Adiabatic regime: Figure 3 shows the sinusoidal potential and the nuclear distributions for all three methods. Even when the energy is not enough to get to the excited adiabatic state, by tuning the coupling so that $\xi \approx 1$ we create a population in the excited state, making EH dynamics slower; whereas SH dynamics will experience rejected hops, following a trajectory on the ground state that reproduces the $\mathrm{SO}$ results accurately.

b. Nonadiabatic regime: Figure 4 shows nuclear distributions for the different coupling regimes. All three methods yield very similar results if Massey's parameter is not near 1. For large and small couplings, the nuclear wave-packet moves either highly adiabatically or diabatically and both $\mathrm{EH}$ and $\mathrm{SH}$ reproduce quantum simulations well (Figs. 4d,f).

Branching of nuclear trajectories will be particularly noticeable for the intermediate coupling case that corresponds to $\xi=1.9$ (Fig. 4a). At every diabatic crossing, the coupling splits the ensemble into two parts, one is following the adiabatic route on the ground state and the other is transferred to the upper state and is reflected by its repulsive part. These competing pathways with different behaviors are not captured well with $\mathrm{EH}$ dynamics (Fig. 4b).

c. High energy regime: Even setting $\xi=1$ does not break down the EH method for the high initial energy case (Fig. 5), all three methods produce similar dynamics. The rationale for this behavior is similarity in slopes of ground and excited PESs over a distance spanning several minima. However, one can notice that the exact distribution has two parts (faster and slower) while the EH distribution is centered right at their separation point. This result hints that at longer times the separation between two parts of the exact distribution may grow while the EH distribution will be approximating their average.

\section{B. Metallic surface model}

To model dynamics on a metallic surface where more electronic states are accessible, we use the model that was originally introduced in Ref. 1 for representing a chemisorbed atom on a 1D metallic chain of atoms. The total diabatic potential is built in three steps. First, an elementary building block is chosen as a harmonic potential describing the chemical bonding in an adatommetal dimer:

$$
V_{0}(R)=\frac{M \Omega^{2} R^{2}}{2},
$$

where $\Omega$ is a harmonic frequency. Second, this elementary potential is replicated by defining $V_{k D}(R)=V_{0}(R-k D)$ for $-n \leq k \leq n$, and all replicas are placed in interacting diabatic potential matrix

$$
V(R)=\left[\begin{array}{cccc}
V_{-n D}(R) & \beta_{1} & \beta_{2} & \ldots \\
\beta_{1} & V_{(-n+1) D}(R) & \beta_{1} & \vdots \\
\vdots & \beta_{1} & \ddots & \\
\cdots & \cdots & & V_{n D}(R)
\end{array}\right]
$$

with $\beta_{i}$ coupling constants, and $D$ the distance between the atoms of the chain. Third, this set of diabatic potentials is replicated $m+1$ times vertically in the energy direction with addition of a small spatial shift $d$ and diabatic coupling $\alpha$ between nearest neighboring replicas. The scalar potential $V_{k D}$ in Eq.(15) is then replaced by a $(m+1) \times(m+1)$ tridiagonal matrix with diagonal elements $\left[\mathbb{V}_{k D d \alpha}\right]_{i, i}(R)=V_{k D}(R+(i-1) d)$, and each coupling constant becomes a $(m+1) \times(m+1)$ diagonal matrix

$$
\mathbf{V}(R)=\left[\begin{array}{ccc}
\mathbb{V}_{-n D d \alpha}(R) & \beta_{1} & \ldots \\
\beta_{1} & \ddots & \vdots
\end{array}\right] .
$$

All parameters used for this model are given in Table 1. To summarize, the metallic model has two types of

TABLE I. Parameters of the metallic surface model

\begin{tabular}{lc}
\hline Parameter & Value (a.u.) \\
\hline Number of atoms in a chain $2 n+1$ & 9 \\
Harmonic frequency $\Omega$ & 0.0028 \\
Atom chain distance $D$ & 5.0 \\
Inter-layer couplings: & \\
$\beta_{1}$ & $0.03,0.011$ \\
$\beta_{2}$ & 0.02 \\
$\beta_{i>2}$ & 0.01 \\
Intra-layer coupling $\alpha$ & 0.004 \\
Intra-layer offset $d$ & $1.0 \times 10^{-4}$ \\
Number of layers $m+1$ & 10 \\
\hline
\end{tabular}

couplings: $\alpha$, between the almost parallel PESs (intralayer), which are to model electron-hole excitations. $\beta_{i}$ between the PESs on different equilibrium positions on the surface (inter-layer), and yield adiabatic PESs with very different slopes, as it can be seen in Fig. 7a.

There are two main energetic regimes which will be of interest. (1) Frictional regime: The initial energy is sufficient to stimulate nonadiabatic transitions between almost parallel PESs constituting the first layer but is insufficient for significant population of an excited adatom 


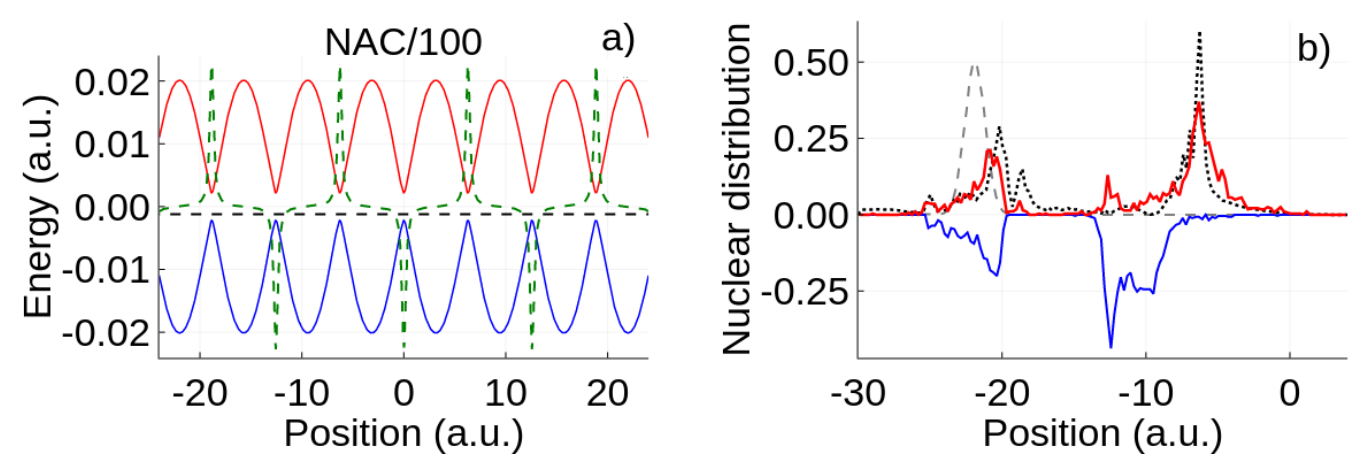

FIG. 3. a) Sinusoidal model with coupling $\Delta=0.0022$ and $\xi=1.5$ : PESs (solid), initial energy (horizontal dashed), and NAC (green dashed). b) Initial (dashed gray, its magnitude divided by 2, the average momentum $p=8.7$ ) and final nuclear positions for nuclear trajectories (blue for $\mathrm{EH}$, red for $\mathrm{SH}$, and black dots for $\mathrm{SO}$ ). EH results were mirrored for clarity.
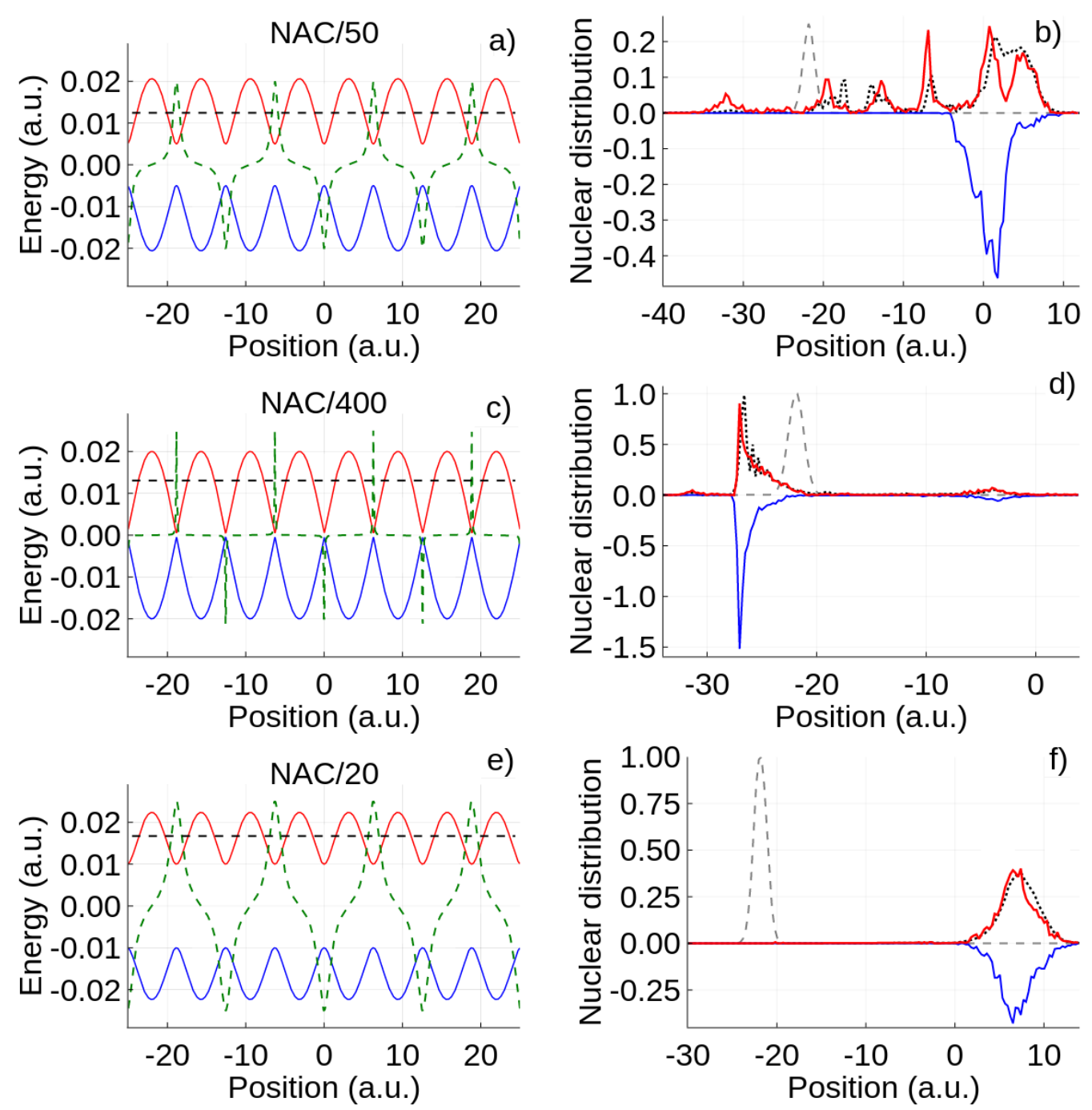

FIG. 4. a), c) and e): PESs (solid), initial energy (horizontal dashed), and NACs (green dashed). b), d) and f): initial (dashed gray, its magnitude divided by 4 for b) and final nuclear distributions (blue for EH, red for SH, and black dots for SO). Diabatic couplings, $\Delta$ : $0.005(\mathrm{~b}), 0.0005(\mathrm{~d})$, and $0.01(\mathrm{f})$. Initial Wigner distributions' average momenta: $p_{0}=11.5(\mathrm{~b}$ and $\mathrm{d})$ and $p_{0}=12.5$ (f). EH results were mirrored for clarity. The adiabatic Massey parameters, $\xi: 1.9$ (b), 0.021 (d), and 6.1 (f). 

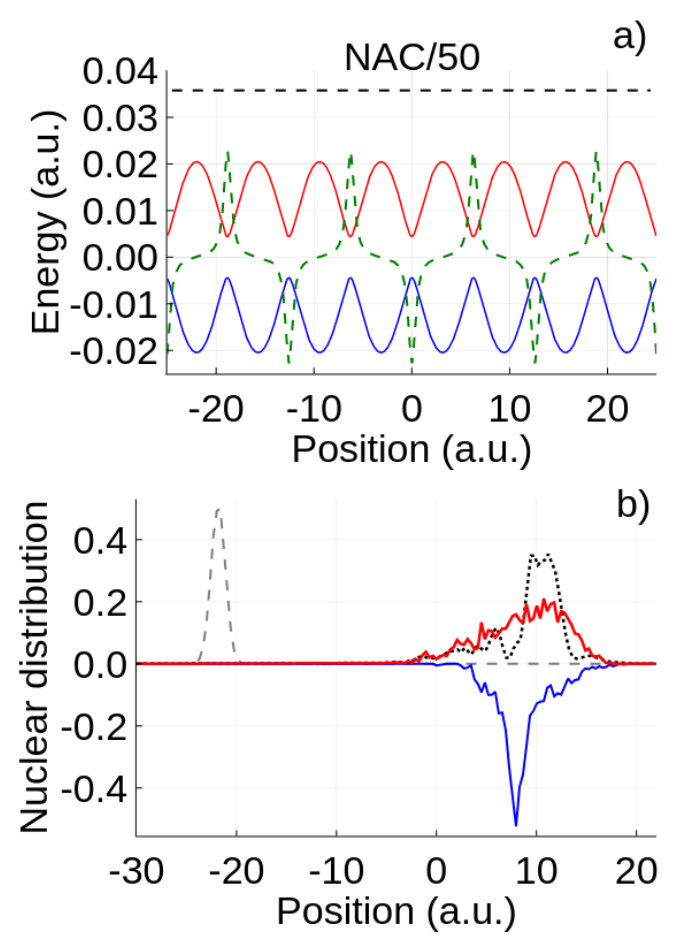

FIG. 5. a) Sinusoidal model with coupling $c=0.0044$ and $\xi=0.96$ : PESs (solid) and initial energy (horizontal dashed). b) Initial nuclear position distribution (dashed gray, its magnitude is divided by 2 , the average momentum $p_{0}=15$ ), and final nuclear position distributions: $\mathrm{EH}$ (blue, mirrored for clarity), $\mathrm{SH}$ (red) and SO (black dots).

state corresponding to the second layer (Fig. 6). (2) Twolayer regime: The initial energy allows the system to have similar probabilities for the first and second layers (Fig. 7). Even higher initial energies are not expected to add new dynamical regimes because excitations to higher layers will only increase possible deviations of dynamics on different layers.

a. Frictional regime: Figure 6 shows the results for an inter-layer coupling of $\beta_{1}=0.03$. Both $\mathrm{EH}$ and $\mathrm{SH}$ yield very similar results: the electronic population has spread over the layered states, and both methods capture most of the nuclear branching. Both methods show a frictional behaviour: Fig. $6 \mathrm{c}$ shows the deviation against a trajectory on the adiabatic ground state (i.e. Born-Oppenheimer dynamics). The adiabatic Massey parameter is $\xi=874$, meaning there are no competing pathways in different layers, which is consistent with the $\mathrm{EH}$ results being almost identical to the SH ones.

b. Two-layer regime: When we used $\beta_{1}=0.03$, we obtained a ballistic-like motion, both $\mathrm{EH}$ and $\mathrm{SH}$ yield dynamics that are extremely similar to the SO ones: we are in an adiabatic regime $(\xi=6.0)$, and $\mathrm{EH}$ is able to model this correctly, including the intra-layer effects. Figure 7 shows the results for the $\beta_{1}=0.011$ regime. As expected, EH fails to account for the branching of the nuclear trajectories, obtaining results that are very different from the exact quantum ones, while SH recovers results that are a lot more accurate. The inter-layer coupling is
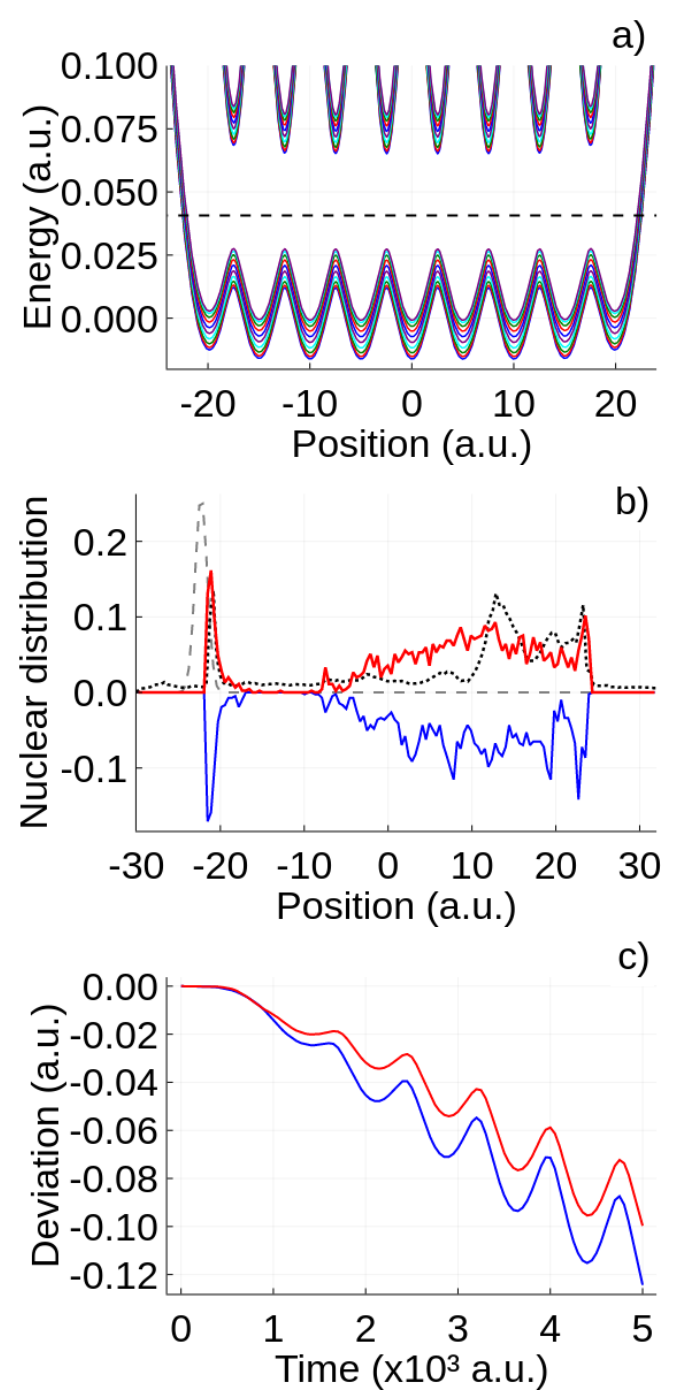

FIG. 6. a) Metallic surface model, $\xi=874$ (between the ground state and the first excited state of the next layer): PESs (solid) and initial energy (horizontal dashed). b) Initial (dashed gray, divided by 4 , zero average momentum) and final nuclear positions for nuclear trajectories (blue for $\mathrm{EH}$, red for $\mathrm{SH}$, and black dots for $\mathrm{SO}$ ). EH results were mirrored for clarity. c) Deviations from a Born-Oppenheimer trajectory with zero initial momentum and -22 a.u. initial position.

then responsible for the breakdown of EH dynamics, and our indicator can be applied for such transitions.

\section{CONCLUSIONS}

We have analyzed break-down of the EH approach in several one-dimensional models containing multiple electronic states and amenable to numerically exact treatment. The main condition for $\mathrm{EH}$ failure is accessibility of several electronic PESs with different nuclear forces. The numerical indicator for identifying such cases, the Massey parameter, has been suggested and assessed. When the Massey parameter is much larger or much smaller than 
a)
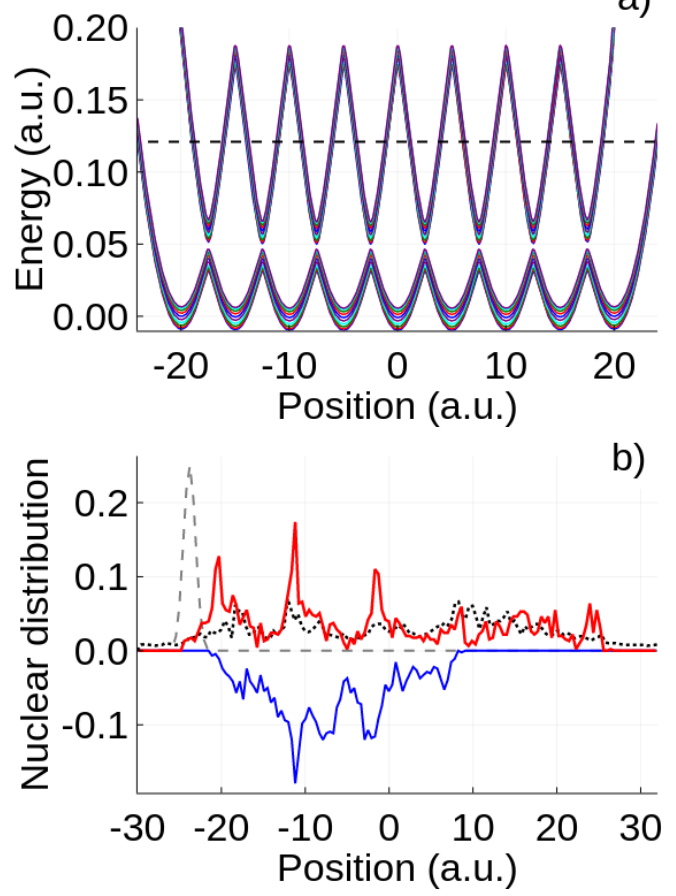

FIG. 7. a) Metallic surface model with inter-layer coupling $\beta_{1}=0.011$ and $\xi=0.90$ (between the ground state and the first excited state of the next layer): PESs (solid), initial energy (horizontal dashed). b) Initial (dashed gray, its magnitude is divided by 4 , zero average momentum) and final nuclear position distributions (blue for $\mathrm{EH}$, red for $\mathrm{SH}$, and black dots for SO). EH results were mirrored for clarity.

$1, \mathrm{EH}$ and SH yield very accurate results. On the other hand, when the Massey parameter approaches 1 for several competing pathways, the EH breaks down failing to simulate nuclear dynamics following one of the competing pathways. SH can properly treat such cases and outperforms EH in modeling dynamics for the considered models. The Massey parameter can be calculated using diabatic [Eq. (10)] or adiabatic parameters [Eq. (11)], and although the two versions are numerically somewhat different, they qualitatively agree in most of the cases. Even though, the considered models were inspired by possible PESs of periodic systems, our results applicable not only to dynamics on surfaces: the given criterion could be applied to any system with nonadiabatic transitions and localized NACs.

Since friction theories are based on the EH approach, the breakdown of $\mathrm{EH}$ will lead to the breakdown of any friction-based method. When the Massey parameter approaches 1 , any method that does not incorporate several possible paths will be unable to properly model the dynamics. This result is consistent with that of Coffman and Subotnik $^{25}$, where it has been concluded that a friction approach seems to fail in the case where there are nonequivalent pathways. Such behavior can be explained by the breakdown of the underlining EH theory as opposed to an intrinsic failure of the friction approach.

\section{ACKNOWLEDGEMENTS}

I.L.G. is grateful to Ilya G. Ryabinkin and Rami Gherib for stimulating discussions. A.F.I. acknowledges the financial support from the Ontario Ministry of Research and Innovation through an Early Researcher Award.

\section{APPENDIX A: THE LANDAU-ZENER MODEL}

Here we reformulate the Massey parameter for the LZ model in the adiabatic quantities. First, let us derive the time-derivative coupling between the adiabatic states of the LZ model. The LZ model potential is a timedependent matrix in the diabatic representation

$$
V(t)=\left[\begin{array}{cc}
\delta t & \Delta \\
\Delta & -\delta t
\end{array}\right]
$$

The eigenfunctions (adiabatic states) of this potential are

$$
\left|\phi_{1}\right\rangle=\left[\begin{array}{c}
\sin \left(\frac{\theta}{2}\right) \\
-\cos \left(\frac{\theta}{2}\right)
\end{array}\right] ;\left|\phi_{2}\right\rangle=\left[\begin{array}{l}
\cos \left(\frac{\theta}{2}\right) \\
\sin \left(\frac{\theta}{2}\right)
\end{array}\right]
$$

where $\tan (\theta)=\Delta /(\delta t)$. The corresponding eigenvalues are $E_{1}=-\sqrt{\delta^{2} t^{2}+\Delta^{2}}$ and $E_{2}=\sqrt{\delta^{2} t^{2}+\Delta^{2}}$, and the time-derivative coupling is

$$
\begin{aligned}
\tau & =\left\langle\phi_{1} \mid \partial_{t} \phi_{2}\right\rangle=\frac{\left\langle\phi_{1}\left|\partial_{t} V\right| \phi_{2}\right\rangle}{\omega} \\
& =\frac{1}{\omega}\left[\sin \left(\frac{\theta}{2}\right)-\cos \left(\frac{\theta}{2}\right)\right]\left[\begin{array}{cc}
\delta & 0 \\
0 & -\delta
\end{array}\right]\left[\begin{array}{c}
\cos \left(\frac{\theta}{2}\right) \\
\sin \left(\frac{\theta}{2}\right)
\end{array}\right] \\
& =\frac{\delta \sin (\theta)}{\omega}
\end{aligned}
$$

where $\omega=E_{2}-E_{1}$. Accounting for $\sin (\theta)=$ $\Delta / \sqrt{\delta^{2} t^{2}+\Delta^{2}}$ and $\omega=2 \sqrt{\delta^{2} t^{2}+\Delta^{2}}$ we obtain

$$
\tau=\frac{\delta \Delta}{2\left(\delta^{2} t^{2}+\Delta^{2}\right)} .
$$

Second, at zero time, when the particle reaches the diabatic intersection point, $\tau$ can be turned into a quantity that will be useful for our purpose by division on $\omega$

$$
\frac{\tau(t=0)}{\omega}=\left.\frac{\delta \Delta}{4\left(\delta^{2} t^{2}+\Delta^{2}\right)^{\frac{3}{2}}}\right|_{t=0}=\frac{\delta}{4 \Delta^{2}} .
$$

Third, let us reformulate the diabatic Massey parameter by calculating the diabatic force difference. We can write the force matrix $F=-\nabla V$, and using the chain rule for the derivative we get

$$
F=\frac{1}{\dot{R}} \frac{\partial V}{\partial t}=\frac{1}{\dot{R}}\left[\begin{array}{cc}
\delta & 0 \\
0 & -\delta
\end{array}\right] .
$$

Therefore the diabatic force difference is given by $\left|F_{b}-F_{a}\right|=2 \delta \dot{R}^{-1}$. Note that the LZ model assumes 
a quick transition through a crossing, meaning the nuclear velocity will not suffer any significant changes: it can be thought of as a constant, a good first order approximation if the force is acting for a short time. Replacing Eq. (24) into the diabatic Massey parameter:

$$
\xi=\frac{2 \pi \Delta^{2}}{\dot{R}\left|F_{b}-F_{a}\right|}=\frac{\pi \Delta^{2}}{\delta} .
$$

Using Eq.(23) we can rewrite this as

$$
\xi=\frac{\pi}{4} \frac{4 \Delta^{2}}{\delta}=\frac{\pi \omega}{4 \tau(t=0)}
$$

obtaining the adiabatic Massey parameter shown in Eq.(11).
${ }^{1}$ I. G. Ryabinkin and A. F. Izmaylov, "Mixed quantumclassical dynamics using collective electronic variables: A better alternative to electronic friction theories," J. Phys. Chem. Lett. 8, 440-444 (2017).

${ }^{2}$ W. Dou and J. E. Subotnik, "A generalized surface hopping algorithm to model nonadiabatic dynamics near metal surfaces: The case of multiple electronic orbitals," J. Chem. Theory Comput. 13, 24302439 (2017).

3 N. Shenvi and J. C. Tully, "Nonadiabatic dynamics at metal surfaces: Independent electron surface hopping with phonon and electron thermostats," Faraday Discuss. 157, 325-335 (2012).

${ }^{4}$ R. Gherib, I. G. Ryabinkin, and A. F. Izmaylov, "Why Do Mixed Quantum-Classical Methods Describe Short-Time Dynamics through Conical Intersections So Well? Analysis of Geometric Phase Effects," J. Chem. Theory and Comp. 11, 1375-1382 (2015).

5 N. L. Doltsinis and D. Marx, "First principles molecular dynamics involving excited states and nonadiabatic transitions," J. Theor. Comput. Chem. 01, 319 (2002).

6 J. C. Tully, "Molecular dynamics with electronic transitions," J. Chem. Phys. 93, 1061 (1990).

7 L. Wang, A. Akimov, and O. V. Prezhdo, "Recent Progress in Surface Hopping: 2011-2015," J. Phys. Chem. Lett. 7, 2100-2112 (2016).

8 N. Shenvi, J. R. Schmidt, S. T. Edwards, and J. C. Tully, "Efficient discretization of the continuum through complex contour deformation," Phys. Rev. A 78, 022502 (2008).

9 N. Shenvi, S. Roy, and J. C. Tully, "Nonadiabatic dynamics at metal surfaces: Independent-electron surface hopping," J. Chem. Phys. 130, 174107 (2009).

10 M. Head-Gordon and J. C. Tully, "Molecular dynamics with electronic frictions," J. Chem. Phys. 103, 10137 (1995).

11 M. Askerka, R. J. Maurer, V. S. Batista, and J. C. Tully, "Role of tensorial electronic friction in energy transfer at metal surfaces," Phys. Rev. Lett. 119, 069901 (2017).

12 R. J. Maurer, B. Jiang, H. Guo, and J. C. Tully, "Mode specific electronic friction in dissociative chemisorption on metal surfaces: $\mathrm{H}_{2}$ on $\mathrm{Ag}(111)$," Phys. Rev. Lett. 118,
256001 (2017).

13 W. Dou, A. Nitzan, and J. E. Subotnik, "Frictional effects near a metal surface," J. Chem. Phys. 143, 054103 (2015).

${ }^{14}$ W. Dou and J. E. Subotnik, "A many-body states picture of electronic friction: The case of multiple orbitals and multiple electronic states," J. Chem. Phys. 145, 054102 (2016).

15 W. Dou and J. E. Subotnik, "Electronic friction near metal surfaces: A case where molecule-metal couplings depend on nuclear coordinates," J. Chem. Phys. 146, 092304 (2017).

16 A. Eisenstein, L. Leung, T. Lim, Z. Ning, and J. C. Polanyi, "Reaction dynamics at a metal surface; halogenation of Cu(110)," Faraday Discuss. 157, 337-353 (2012).

17 L. Leung, T. Lim, Z. Ning, and J. C. Polanyi, "Localized reaction at a smooth metal surface: p-diiodobenzene at Cu(110)," J. Am. Chem. Soc. 134, 9320-9326 (2012).

18 I. Loaiza and A. F. Izmaylov, Surface hopping with collective electronic variables (in preparation).

19 J. C. Tully, "Mixed quantum-classical dynamics," Faraday Discuss. 110, 407-419 (1998).

20 D. J. Tannor, Introduction to Quantum Mechanics (University Science Books, 2007) Chap. 11.7.

21 C. Zener, "Non-adiabatic crossing of energy levels," Proc. R. Soc. Lon. A 137, 696-702 (1932).

${ }^{22}$ H. S. W. Massey, "Collisions between atoms and molecules at ordinary temperatures," Rep. Prog. Phys. 12, 248 (1949).

23 C. De Grandi and A. Polkovnikov, "Adiabatic perturbation theory: From Landau-Zener problem to quenching through a quantum critical point," in Quantum Quenching, Annealing and Computation (Springer Berlin Heidelberg, 2010) pp. $75-114$.

24 M. D. Hack, A. W. Jasper, Y. L. Volobuev, D. W. Schwenke, and D. G. Truhlar, "Do semiclassical trajectory theories provide an accurate picture of radiationless decay for systems with accessible surface crossings?" J. Phys. Chem. A 104, 217232 (2000).

25 A. J. Coffman and J. E. Subotnik, "When is electronic friction reliable for dynamics at a molecule-metal interface?" Phys. Chem. Chem. Phys. 20, 9847-9854 (2018). 